\title{
The property diagram in heat transfer and its applications
}

\author{
CHEN Qun*, XU YunChao \& GUO ZengYuan \\ Key Laboratory for Thermal Science and Power Engineering of Ministry of Education, Department of Engineering Mechanics, Tsinghua \\ University, Beijing 100084, China
}

Received May 12, 2012; accepted August 21, 2012

\begin{abstract}
Inspired by the property diagrams in thermodynamics, which distinctly reflect the performance and characteristics of thermodynamic cycles, we establish a state equation for heat motion and introduce a two-dimension property diagram, $T$ - $q$ diagram, in heat transfer to analyze and optimize the performance of heat exchangers, where heat flow is a state parameter for heat motion. According to the property diagram, it is convenient to obtain the influences of heat exchanger area, heat capacity rate and flow arrangement on the heat transfer performance during the analysis of heat exchangers and their networks. For instance, when analyzing the heat exchanger network in a district heating system, it is obvious to find that: if both the heat demand and the indoor air temperature in each branch of the network are the same, the total area of heat exchangers, the flow rate of water and the return water temperature in each branch are all the same; if the indoor air temperatures in different branches are different, the temperatures of the waters after flowing through different branches are different, which means that the mixing process of return waters with the same temperature is not an essential requirement to realize the best performance of district heating systems.
\end{abstract}

heat transfer, property diagram, irreversibility, entransy dissipation, optimal design

Citation: $\quad$ Chen Q, Xu Y C, Guo Z Y. The property diagram in heat transfer and its applications. Chin Sci Bull, 2012, 57: 4646-4652, doi: 10.1007/s11434-012$5476-5$

Heat is one of the most common forms during energy utilization [1-4]. It can be obtained from the chemical energy of fuels through combustion, the atomic energy of radioactive material by either nuclear fission or nuclear fusion, and the solar energy or the geothermal energy by direct collection. Meanwhile, there are two different purposes in heat utilization: one is to generate power, and the other is to directly heat or cool objects. In the former purpose, heat is converted to mechanical energy through the heat absorption, expansion, heat release and compression processes of working substances, where the property diagrams in thermodynamics, e.g. $p-v, T-s$ and $h-s$ diagrams, are wildly used to directly express the variation rules of the state parameters of the working substances during a heat-work conversion process, qualitatively describe the exchanges of work and heat between the working substances and the surrounding environment in a thermodynamic cycle, and optimal design some ideal thermodynamic cycles for promoting the con-

*Corresponding author (email: chenqun@tsinghua.edu.cn) version efficiency.

A thermodynamically reversible cycle is composed of several quasi-equilibrium processes, i.e. quasi-static processes, which have to progress at an infinitely slow rate in order to be reversible. Therefore, the output power of an ideal reversible thermodynamic cycle is zero. That is, a reversible cycle is a theoretical construct, which cannot actually occur and is seldom even approached in reality. In practical thermodynamic cycles, all real processes take place with finite potential difference and have finite rates, so they are all irreversible, where the property diagram in thermodynamics cannot be directly used to analyze and optimize the performance. For instance, heat transfer processes with finite temperature difference are irreversible processes and heat is regarded as a process parameter in thermodynamics, so the property diagram cannot be constructed in heat transfer to analyze and optimize the performance.

In order to analyze heat transfer with finite temperature difference, Guo et al. [5] introduced a new physical quantity, entransy, to describe the ability of an object transferring 
heat to the other object and used entransy dissipation to measure the irreversibility of heat transfer. This has already been successfully applied to analyze and optimize heat and mass transfer processes and apparatus including heat conduction [5-8], heat convection [9-14], thermal radiation [15], evaporative cooling [16,17] and heat exchanger (networks) [18-23]. In this article, we briefly review the origin and applications of the property diagrams in thermodynamics, propose two state parameters in heat transfer based on the analogy between heat transfer and fluid flow, construct a property diagram in heat transfer by taking the two state parameters as coordinates to describe the variations of entransy for both hot and cold fluids during heat transfer processes and meanwhile measure the irreversibility of the processes, and finally to optimize heat transfer performance and promote energy utilization efficiency qualitatively.

\section{Property diagrams in thermodynamics}

In thermodynamics, the parameters that describe the equilibrium state of the working substances are called state parameters, such as pressure, temperature and entropy. Moreover, the stable equilibrium state of a closed simple compressible system with given internal restraints can be characterized completely by only two independent state parameters. For example, the two independent parameters, pressure and specific volume, can determine the equilibrium state of ideal gas. Taking any two independent state parameters to be the $x$ and $y$ coordinates, the constructed plane is property diagrams in thermodynamics, e.g. the $p-v$ and $T-s$ diagrams showed in Figure 1. In the property diagrams, each point represents an equilibrium state of the substances and each curve represents a quasi-equilibrium process, where the relation between the state and process parameters is described based on the first law of thermodynamics as:

$$
\mathrm{d} U=\delta Q-\delta W,
$$

where $U$ represents the thermodynamic energy of the substances, $Q$ and $W$ respectively stand for the heat and work exchanged between the substances and the environment. In eq. (1), the thermodynamic energy is a state parameter which has the total differential form, $\mathrm{d} U$, to describe its variation. However, the heat and work exchanged are all process parameters which have to be represented by $\delta Q$ and $\delta W$, respectively, and can be clearly expressed by the area which are surrounded by the process curves, and the horizontal and vertical axes. For example, in Figure 1(a), the shaded area under the process curve (1-2) stands for the work of expansion, while the shaded area towards the vertical axis of the process curve (1-2) represents the flow work exchanged between substances and environments. In Figure 1(b), the shaded area under the process curve (1-2) is the heat exchanged between substances/systems and environments in reversible processes. Therefore, through calculat-
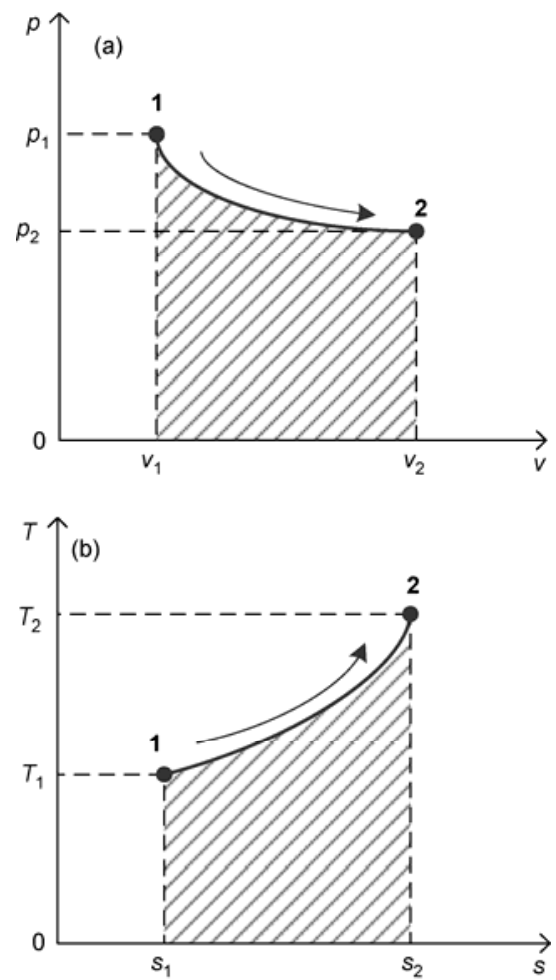

Figure 1 The property diagrams in thermodynamics. (a) $p-v$ diagram; (b) $T-s$ diagram.

ing the shaded area, the expansion work, $w$, flow work $w_{t}$ and heat $q$ can be easily obtained [1-4].

$$
w=\int_{1}^{2} p \mathrm{~d} v, w_{t}=-\int_{1}^{2} v \mathrm{~d} p, q=\int_{1}^{2} T \mathrm{~d} s,
$$

where $s$ is the specific entransy.

Moreover, in a property diagram, the closed polygon constructed by some process curves directly depicts a thermodynamic cycle. For example, Figure 2(a) and 2(b) separately show the four thermodynamic processes in the reversible Carnot cycle, i.e. the isothermal heat absorption process (1-2), the adiabatic expansion process (2-3), the isothermal heat rejection process (3-4) and the adiabatic compression process (4-1), respectively in the $p-v$ and $T-S$ diagrams. In Figure 2(a) and (b), the shaded area surrounded by the four process curves separately represent the mechanical work and the heat exchanged between the systems and the surrounding environments in the Carnot cycle.

\section{Property diagrams in heat transfer}

Heat transfer is thermal energy in transit due to a temperature difference [24], which cares about how to control (increase or reduce) the heat transfer rate with a given temperature difference or how to reduce the temperature difference when the heat transfer rate is fixed. Because heat, in classic thermodynamics, is a process parameter, there is no property diagram constructed with heat as the horizontal or vertical 

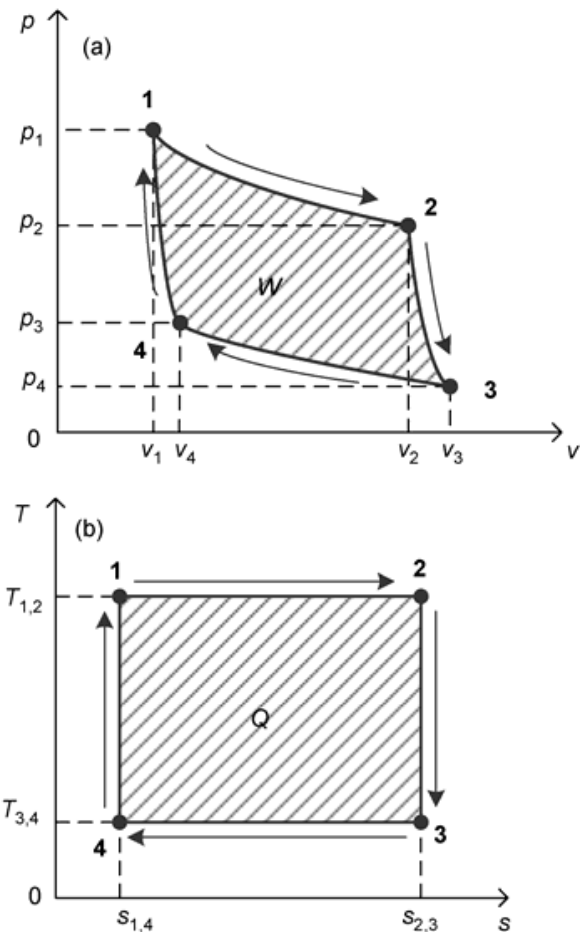

Figure 2 The property diagrams of reversible Carnot cycle. (a) $p-v$ diagram; (b) $T$-s diagram.

coordinate to analyze heat transfer performance. Instead, the common diagram to analyze the fluid temperature variations in a heat exchanger is the $T$ - $A$ diagram, which is constructed with the temperature, $T$, and the heat exchange area, $A$, separately as the vertical and horizontal coordinates. As shown in Figure 3, the temperature of the hot fluid falls from $T_{h, \text { in }}$ at the inlet to $T_{h, \text { out }}$ at the outlet and that of the cold fluid raises from $T_{c, \text { in }}$ at the inlet to $T_{c \text {,out }}$ at the outlet. Meanwhile, on both sides of the shaded area $\mathrm{d} A$, the temperatures of the hot and cold fluids are $T_{h}$ and $T_{c}$, respectively, and hence the heat flux, $\mathrm{d} q$, between the hot and cold fluids is

$$
\mathrm{d} q=K\left(T_{h}-T_{c}\right) \mathrm{d} A,
$$

where $K$ is the heat transfer coefficient of the heat exchang-

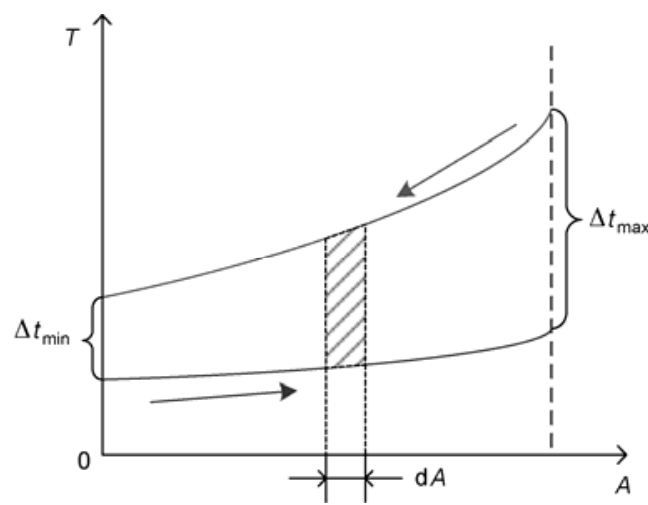

Figure 3 The variations of fluid temperatures with the heat transfer area in a counter-flow heat exchanger. ers. Integral of eq. (3) offers the total heat transferred between the hot and cold fluids in the heat exchangers, which corresponds to the area of the quadrilateral in Figure 3.

Although the quadrilateral in the $T$ - $A$ diagram can reflects heat transfer performance in a heat exchanger, the area $A$ is not a state parameter of fluids, and thus the areas under the temperature curves of both hot and cold fluids has no clear physical meaning. Most importantly, the $T$ - $A$ diagram is not the property diagrams in heat transfer and cannot reveal the irreversibility of heat transfer.

In particle dynamics, velocity and location determine the state of moving particles, which means that velocity and location are the state parameters for particles motion. In fluid mechanics, pressure, velocity, acceleration, momentum and kinetic energy are all the state parameters to describe the state of fluid flow. All of these state parameters satisfy the related governing equations. Moreover, it should be addressed that because any state parameter of the system corresponds to only one process parameter with same characteristic, there is no need to distinguish the state and process parameters in fluid mechanics, e.g. the dissipation of mechanical energy equivalent to the generation of thermal energy.

For the fully-developed laminar flow in a regular and straight horizontal circular tube, there is no net mass importing or exporting in each differential control volume but the transfer of kinetic and potential energy among adjacent differential control volumes or walls due to the drive of velocity gradient. Because the potential energy will dissipate to the thermal energy due to viscous effect, the pressure of fluid drops along the flow direction. In this case, the governing equation to describe the relation between pressure and velocity is

$$
\mathrm{d} p=\frac{8 \mu}{R} u \mathrm{~d} L
$$

where $\mu$ is the viscosity, $R$ is the radius of the circular tube, $u$ is the average velocity of the fluids, $L$ is the distance.

Taking the pressure $p$ and the volume flow rate $\dot{V}$, the two independent state parameters describing fluid flow, as the horizontal and vertical coordinates, we can construct the property diagrams, as shown in Figure 4, in fluid mechanics. Any point in the diagram represents a state of fluid flow and the area under the process curve (1-2) stands for the mechanical energy dissipation during fluid flow. That is, by calculating the shaded area, the dissipation rate of mechanical energy during the fluid flow process in the circular tube, $\Phi_{m}$, is easily obtained.

$$
\Phi_{m}=\dot{V} \Delta p
$$

Heat transfer and fluid flow are two analogous physical phenomena. However, in the existing heat transfer studies, there is no physical quantity to describe the motion state of heat, e.g. the velocity and the potential of heat, and it lacks a 


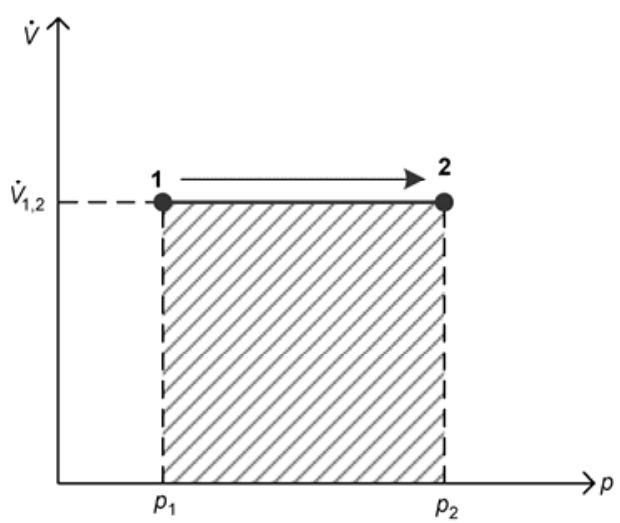

Figure 4 The variation of the fluid pressure with the volume flow rate in a regular straight horizontal circular tube.

state equation in heat transfer, so it is difficult to establish a property diagram for the analysis of heat transfer.

For a fluid flow process with the mass flow rate $\dot{m}$, specific heat capacity, $c_{p}$ and temperature $T$, its enthalpy flow rate is:

$$
\dot{h}=\dot{m} c_{p} T \text {. }
$$

After flowing through a certain heat transfer area, the temperature variation of the fluid is $\Delta T$, and its corresponding heat flow, $q$, that the fluid absorbs or releases is:

$$
q=\dot{m} c_{p} \Delta T .
$$

According to the thermomass theory [25], heat transfer are the flow processes of thermomass. Meanwhile, the related flow velocity of thermomass during heat transfer, $u_{h}$, is expressed as

$$
u_{h}=\frac{q}{\rho C T A},
$$

where $\rho$ and $C$ are the density and the specific heat capacity of heat transfer medium, respectively.

In fluid mechanics, velocity is the state parameter to describe fluid flow. Therefore, the velocity of thermomass is the state parameter to describe thermomass flow, i.e. heat transfer. Moreover, according to the relation between $q$ and $u_{h}$ in eq. (8), heat flow $q$ is also the state parameter to describe the transfer speed of heat. At the same time, eq. (7) is the state equation to reveal the relation among the temperature, the specific heat capacity, the mass flow rate and the heat flow during the heat transfer processes in heat exchangers.

Taking the two independent state parameters, the temperature $T$ and the heat flow $q$, as the horizontal and vertical coordinates as shown in Figure 5, we will obtain the property diagram in heat transfer. Any point in the diagram represents a state of heat flow. The process curves (1-2) and (1-3) represent the heat flow processes of fluids and the slopes of curves are inversely proportional to the heat capacity rates of fluids. Especially, when the heat capacity

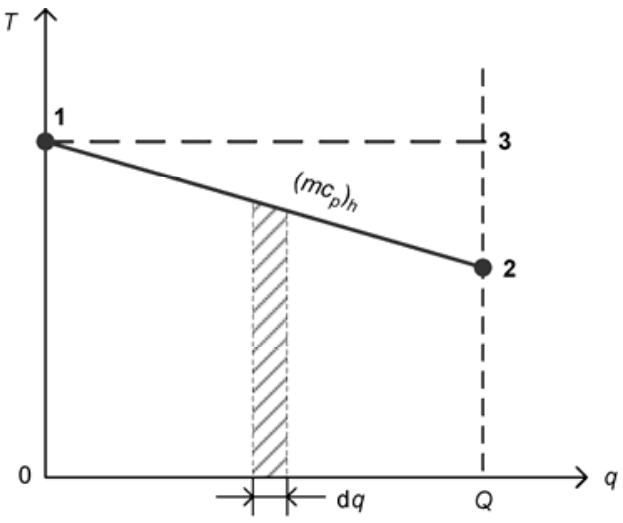

Figure 5 The property diagram in heat transfer.

rate of fluid is infinite, the slope of the process curve approaches zero, i.e. process curve (1-3).

Accompanying with the heat flow, there are also entransy flow during a heat transfer process. For example, when the temperature drop of the fluid is $\mathrm{d} T$ and the corresponding heat flow is $\mathrm{d} q$, the output entransy is $[5,21]$ :

$$
\mathrm{d} \dot{G}=T \mathrm{~d} q,
$$

which can be represented by the shaded area in Figure 5, where the area under the process curve stands for the entransy flow when the fluid temperature drops from $T_{\text {in }}$ to $T_{\text {out }}$, i.e.

$$
\dot{G}=\frac{\left(T_{\text {in }}+T_{\text {out }}\right) q}{2}=\frac{\dot{m} c_{p}\left(T_{\text {in }}^{2}-T_{\text {out }}^{2}\right)}{2} .
$$

When $T_{\text {out }}$ vanishes, the entransy flow exporting from the fluid to the surrounding environment is the maximum. In this case, eq. (10) describes the capacity of the fluid to transfer heat to the environment with the absolute zero of temperature as the benchmark, which is termed entransy:

$$
\dot{G}=\frac{1}{2} \dot{m} c_{p} T_{\text {in }}^{2} .
$$

Figure 6 shows the property diagram for both hot and cold fluids in a two-flow heat exchanger. Through any differential heat transfer area, the heat released from the hot fluid is the same as that absorbed by the cold fluid:

$$
\mathrm{d} q=-\dot{m}_{h} c_{p, h} \mathrm{~d} T_{h}=\dot{m}_{c} c_{p, c} \mathrm{~d} T_{c},
$$

and the shaded area in Figure 6 is

$$
\mathrm{d} S=T_{h} \mathrm{~d} q-T_{c} \mathrm{~d} q,
$$

where the first and second terms on the right side represent the entransy flows that the hot fluid releases and the cold fluid absorbs, respectively. Therefore, the shaded area in Figure 6 stands for the entransy dissipation due to the heat flow, $\mathrm{d} q$, between the hot and cold fluids:

$$
\mathrm{d} \varphi_{h}=\left(T_{h}-T_{c}\right) \mathrm{d} q .
$$




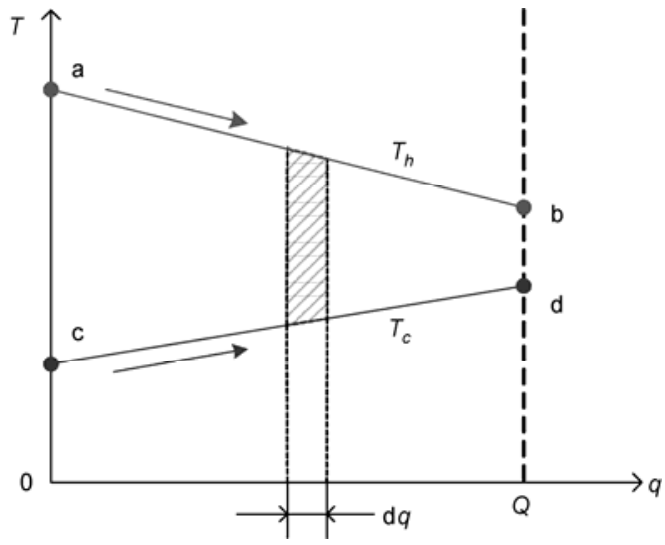

Figure 6 The property diagram for the hot and cold fluids in a heat exchanger.

The area between the process curves $(a-b)$ and $(c-d)$ represent the total entransy dissipation rate in the whole heat exchanger, which can be used to analyze the irreversibility of heat transfer processes and consequently evaluate the heat transfer performance in the heat exchangers:

$$
\Phi_{h}=\int \mathrm{d} \varphi_{h}=\Delta T_{\mathrm{AM}} Q,
$$

where $\Delta T_{\mathrm{AM}}$ is the arithmetic mean temperature difference.

\section{The applications of the property diagram in heat transfer}

\subsection{The analysis of heat transfer performance in heat exchangers}

In heat exchangers with random flow arrangement, the hot fluid with a certain heat capacity rate, $m_{h} c_{p, h}$, needs to be cooled from $T_{h, \mathrm{a}}$ to $T_{h, \mathrm{~b}}$. In Figure 7, the straight line ab indicates the temperature variation of the hot fluid versus the heat flow in the heat exchanger. If the heat exchanger is a counter-flow heat exchanger with infinite heat transfer area and the heat capacity rate of the cold fluid is equivalent to that of the hot fluid, the straight line cd shows the temperature variation of the cold fluid versus the heat flow. In this case, lines cd and ab are coincident, which means the entransy flow that the hot fluid exports equals to that the cold fluid imports. Therefore, there is no entransy dissipation and the heat transfer process in the heat exchanger is reversible.

When the heat transfer area of the heat exchangers is not large enough, the straight line ef in Figure 7 reveals the temperature variation of the cold fluid versus the heat flow in the heat exchanger. Due to the temperature difference between the hot and cold fluids, the entransy flow exported from hot fluid will partly dissipate, which can be directly indicated by the parallelogram abef constructed by lines ab and ef. In this case, the area of abef reflects one of the factors influencing the performance of heat exchangers, i.e. finite heat transfer area.
When the heat capacity rates of hot and cold fluids are different, e.g. $m_{h} c_{p, h}<m_{c} c_{p, c}$, the straight line eg in Figure 7 represents the temperature variation of the cold fluid with a larger heat capacity rate versus the heat flow. As shown in Figure 7 , the difference between the heat capacity rates of hot and cold fluids causes that the area of the trapezoid abeg is larger than that of the parallelogram abef, which means the entransy dissipation during heat transfer increases. The increase of entransy dissipation can be represented by the area of the triangle efg. In this case, increasing the difference of heat capacity rates between the hot and cold fluids will further enlarge the area of triangle efg, i.e. promote the entransy dissipation in the heat exchanger, which reduces the performance of the heat exchanger. Therefore, the area of the triangle efg reflects the second influence factors of the performance of heat exchangers, i.e. the difference of heat capacity rates between two fluids.

Furthermore, when the heat exchanger is parallel-flow, the straight line hi in Figure 7 is the temperature variation of the cold fluid versus the heat flow. In order to cool the hot fluid from $T_{h, \mathrm{a}}$ to $T_{h, \mathrm{~b}}$ with the same heat transfer area, the inlet temperature of the cold fluid drops from $T_{c, \mathrm{e}}$ to $T_{c, \mathrm{~h}}$, which makes the area of the trapezoid abih be larger than that of the trapezoid abef. It means that when the heat capacity rates of the hot and cold fluids in different heat exchangers are the same, parallel-flow heat exchangers have larger entransy dissipation than counter-flow heat exchangers, which is described by the difference between the area of triangles fhk and eki. Therefore, the difference between the area of triangles fhk and eki reflects that the flow arrangement of heat exchangers is another influence factor of the performance of heat exchangers.

In a word, $T-q$ diagram clearly indicates the above three influence factors of the performance of heat exchangers, i.e. finite heat transfer area, difference of heat capacity rates and flow arrangement of heat exchangers.

\subsection{The optimal design of heat exchanger networks}

Figure 8 gives the sketch of a typical heat exchanger net-

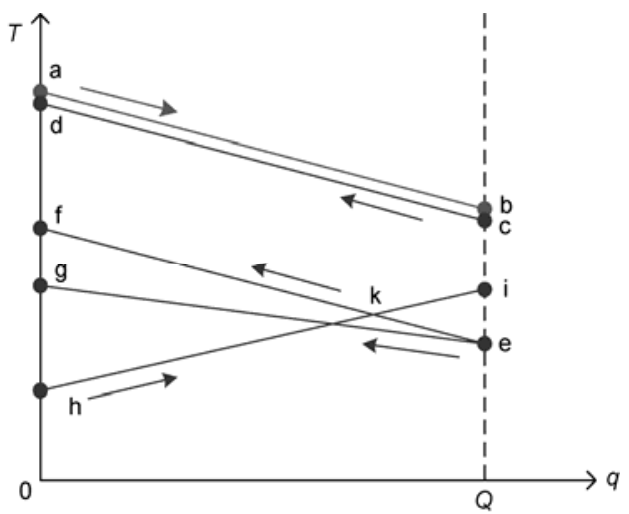

Figure 7 The property diagram for different types of heat exchangers with different heat capacity rates of fluids. 


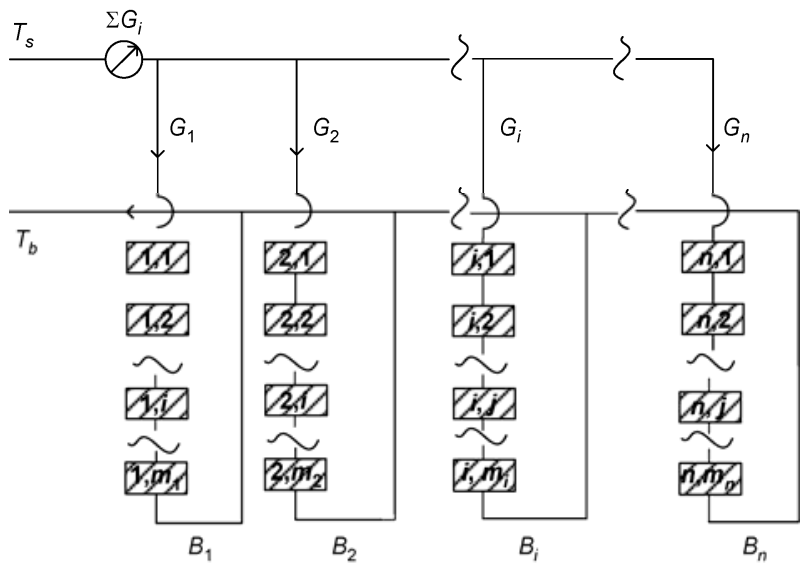

Figure 8 The sketch of a typical heat exchanger network for district heating [22].

work for district heating. The hot water with the inlet temperature, $T_{h \text {,in }}$, and the total mass flux, $m_{\text {in }}$, is distributed into different branches, flows in order through the seriesconnected heat exchangers of the users, satisfies the heat demands of each user, gathers together in the main loop, and finally flows back to the substation. When both the indoor temperature, $T_{i j}$, and the heat demand, $Q_{i j}$ of each users in a branch are all the same, Figure 9 shows the temperature variations of the hot water and the indoor air versus the heat flow, where the heat capacity rate of the indoor air is supposed to be infinite due to the constant indoor air temperature. As shown in Figure 9, along the flow direction of the hot water in each branch, the temperature difference between the hot water and the indoor air decreases, which means the entransy dissipations in downstream heat exchangers becomes less. That is, in order to satisfy the same heating demand, the heat transfer areas of the downstream heat exchangers should be larger, which are approximately inversely proportional to the entransy dissipations in each heat exchanger.

When the indoor temperature, $T_{i j}$, of each user in the heating network and the total heat demand in each heating

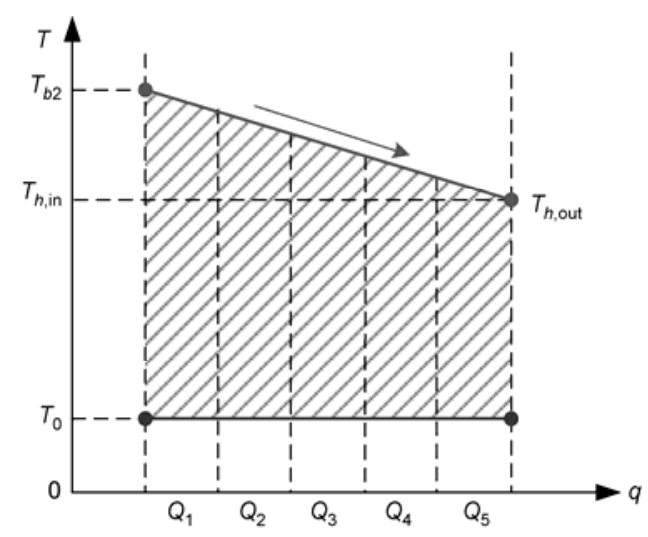

Figure 9 The property diagram for the hot water and the indoor air in a branch. branch, $\sum_{j} Q_{i j}=Q_{i}$, are all the same, Figure 10(a) shows the temperature variations of the hot water and the indoor air versus the heat flow in the heating network with two branches. Due to the symmetry, the total heat transfer areas, the mass flow rates of hot water and the return water temperatures in each branch are the same. If the indoor air temperatures of the two branches are different $\left(T_{1}<T_{2}\right)$, Figure 10(b) shows the temperature variations of the hot water and the indoor air versus the heat flow in each branch. In order to satisfy the heating demand, the mass flow rate of hot water in the second branch needs to increase. If the total mass flow rate of hot water in the network is fixed, the mass flow rate of hot water in the first branch will decrease, which means the total entransy dissipation in the system should be reduced, and the heat transfer areas in both branches need to be enlarged to satisfy the users' demands. Moreover, as shown in Figure 10(b), when the indoor air temperatures are different, the temperatures of the hot waters after flowing through each branch are also different, which means the return waters mixing with the same temperature is not a requirement to obtain the optimal design of district heating networks.

\section{Conclusions}

Inspired by the superior of the property diagram in thermodynamics for the analysis of thermodynamic cycles, we proposes that heat flow is a state parameter in heat transfer, introduces the governing equation, develops a $2 \mathrm{D}$ property diagram in heat transfer, $T-q$ diagram, which can clearly analyze the performance of heat transfer processes. For example, it illustrates the influences of such three factors
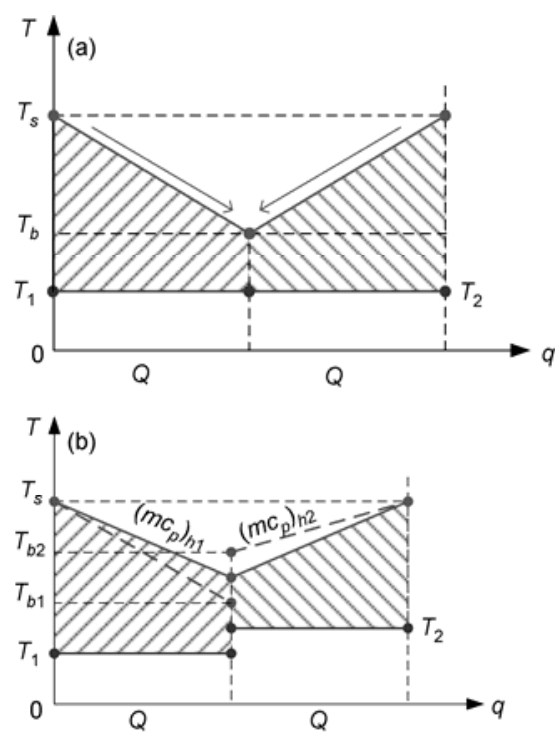

Figure 10 The property diagrams for the hot water and the indoor air in a district heating network with two branches. (a) $T_{1}=T_{2}$; (b) $T_{1} \neq T_{2}$. 
as finite heat transfer area, different heat capacity rates of fluids and flow arrangement on the irreversibility of the heat transfer processes in heat exchangers, which contributes to the optimal design of heat exchanges and their systems.

Furthermore, for the series-connected heat exchanger networks in a district heating system, $T-q$ diagram reflects that along the flow direction of the hot water in each branch, the heat transfer areas of the downstream heat exchangers should be enlarged to reduce the entransy dissipation. For a parallel-connected network with many branches, when both the indoor temperatures of each user and the total heat demands in each heating branch are the same, the total heat transfer areas, the mass flow rates and the return temperatures of the hot waters in each branch are all the same. However, when the indoor air temperatures in different branches are different, the temperatures of the hot water after flowing through the heating branches are not the same, which means the return waters mixing with the same temperature is not a requirement to obtain the optimal design of district heating networks.

In summary, the property diagram in heat transfer contributes to deepen the understanding of heat transfer phenomena and processes and promote the development of heat transfer. In engineering, the property diagram is suitable for measuring the irreversibility and evaluating the performance of heat transfer processes. Furthermore, it can be applied in the optimal designs of heat transfer processes, heat exchangers, heat exchanger networks and different types of complicated energy systems in the future.

This work was supported by the National Natural Science Foundation of China (51006060 and 51036003) and the Foundation for the Author of National Excellent Doctoral Dissertation of China.

1 Zeng D L, Ao Y, Zhang X M, et al. Engineering Thermodynamics (in Chinese). 3rd ed. Beijing: Higher Education Press, 2002

2 Zhu M S, Liu Y, Lin Z Z, et al. Engineering Thermodynamics (in Chinese). Beijing: Tsinghua University Press, 1995

3 Shen W D, Jiang Z M, Tong J G, et al. Engineering Thermodynamics (in Chinese). 3rd ed. Beijing: Higher Education Press, 2001

4 Liu G Y, Liu Z G, Yin J M, et al. Engineering Thermodynamics (in Chinese). Beijing: Higher Education Press, 1998

5 Guo Z Y, Zhu H Y, Liang X G. Entransy-A physical quantity describing heat transfer ability. Int J Heat Mass Transf, 2007, 50: 25452556
6 Chen L G, Wei S H, Sun F R. Constructal entransy dissipation minimization for 'volume-point' heat conduction. J Phys D-Appl Phys, 2008, 41: 195506

7 Chen Q, Zhu H Y, Pan N, et al. An alternative criterion in heat transfer optimization. Porc R Soc A-Math Phys Eng Sci, 2011, 467: 10121028

8 Chen L G, Wei S H, Sun F R. Constructal entransy dissipation rate minimization of a disc. Int J Heat Mass Transf, 2011, 54: 210-216

9 Meng J A, Liang X G, Chen Z J, et al. Experimental study on convective heat transfer in alternating elliptical axis tubes. Exp Therm Fluid Sci, 2005, 29: 457-465

10 Chen Q, Ren J X, Meng J A. Field synergy equation for turbulent heat transfer and its application. Int J Heat Mass Transf, 2007, 50: 5334-5339

11 Chen Q, Ren J X. Generalized thermal resistance for convective heat transfer and its relation to entransy dissipation. Chin Sci Bull, 2008, 53: 3753-3761

12 Chen Q, Wang M R, Pan N, et al. Optimization principles for convective heat transfer. Energy, 2009, 34: 1199-1206

13 Chen Q, Meng J A. Field synergy analysis and optimization of the convective mass transfer in photocatalytic oxidation reactors. Int $\mathbf{J}$ Heat Mass Transf, 2008, 51: 2863-2870

14 Liu W, Liu Z C, Jia H, et al. Entransy expression of the second law of thermodynamics and its application to optimization in heat transfer process. Int J Heat Mass Transf, 2011, 54: 3049-3059

15 Cheng X T, Liang X G. Entransy flux of thermal radiation and its application to enclosures with opaque surfaces. Int J Heat Mass Transf, 2011, 54: 269-278

16 Chen Q, Yang K D, Wang M, et al. A new approach to analysis and optimization of evaporative cooling system I: Theory. Energy, 2010, 35: 2448-2454

17 Chen Q, Pan N, Guo Z Y. A New approach to analysis and optimization of evaporative cooling system II: Applications. Energy, 2011, 36: 2890-2898

18 Liu X, Guo Z Y. A novel method for heat exchanger analysis. Acta Phys Sin, 2009, 58: 4766-4771

19 Chen L, Chen Q, Li Z, et al. Optimization for a heat exchanger couple based on the minimum thermal resistance principle. Int $\mathbf{J}$ Heat Mass Transf, 2009, 52: 4778-4784

20 Qian X D, Li Z, Li Z X. Entransy-dissipation-based thermal resistance analysis of heat exchanger networks. Chin Sci Bull, 2011, 56: 3289-3295

21 Chen Q, Xu Y C. An entransy dissipation-based optimization principle for building central chilled water systems. Energy, 2012, 37: 571579

22 Xu Y C, Chen Q. An entransy dissipation-based method for global optimization of district heating networks. Energ Buildings, 2012, 48: 50-60

23 Yuan F, Chen Q. A global optimization method for evaporative cooling system based on the entransy theory. Energy, 2012, 42: 181-191

24 Incropera F, Dewitt D. Introduction to Heat Transfer. New York: John Wiley \& Sons Inc., 1985

25 Cao B Y, Guo Z Y. Equation of motion of phonon gas and nonFourier heat conduction. J Appl Phys, 2007, 102: 053503

Open Access This article is distributed under the terms of the Creative Commons Attribution License which permits any use, distribution, and reproduction in any medium, provided the original author(s) and source are credited. 